\title{
La Sociopathie \\ de « Louise » \\ dans Chanson Douce
}

\section{par \\ Dr. Abir Chéhata*}

Chanson Douce de Leila Slimani S'ouvre sur un choc terrible, celui du meurtre de deux enfants en bas-âge Milan et Adam par leur nourrice Louise et sa tentative de se suicider.

Dans notre recherche, nous analysons de plus près le personage de la nourrice Louise. Elle est sociopathe, c'est à dire une personne affectée par des troubles de la personnalité manifestant un comportement antisocial. Ce personage complexe et ambigu dissimule un caractère mélancolique.Or cette mélancolique accompagnée d'une solitude déchirante qui s'allonge de plus en plus de façon à devenir une sorte d'escapade des problèmes familiaux et financiers pesant sur sa vie avec le couple.

Des idées absurdes, incongrues la hantent, elle se laisse descendre, peu à peu aux abîmes, dans les méandres de la folie.

Chanson Douce est une histoire réaliste tellement comtemporaine parce qu'elle aborde le statut des nourrices, ébranle les parents et fera vivre d'une autre vision leurs relations avec leurs enfants.

* Maître de conférences -Département de langue et de littérature françaises -Université de Damiette

(La Sociopathie de «Louise » dans Chanson Douce) Dr. Abir Chéhata 
«(...)Nous ne serons heureux (...) que lorsque nous n'aurons plus besoin les uns des autres. Quand nous pourrons vivre une vie à nous, une vie qui nous appartienne, qui ne regarde pas les autres. Quand nous serons libres.»

Slimani (Leila), Chanson Douce, Éditions Gallimard 2016, p.49.

\section{Introduction}

Leila Slimani est L' un des esprits génétiques jalonnant le parcours créatif dans le monde littéraire maghrébin.

Elle est née le 3 octobre 1981 dans une famille marocaine appartenant à un milieu aisé. Son père est un haut fonctionnaire marocain, sa mère, franco-marocaine, est médecin. Fournie de son baccalauréat obtenu au lycée français de Rabat, elle rejoint Paris et le lycée Fénelon pour commencer une classe préparatoire littéraire. Diplômée de l'Institut d'Études Politiques de Paris et après s'être essayée au métier de comédienne, elle se forme au journalisme à l'Express, avant d'être engagée au journal Jeune Afrique en 2008. En2012, elle décide de se consacrer à l'écriture littéraire.

Elle a publié ses deux romans aux éditions Gallimard. Le premier intitulé Dans le jardin de l'ogre, publié en 2014, récompensé du prix littéraire de la Mamounia 2015 et le second, Chanson Douce, publié en 2016, récompensé du prix Goncourt 2016, du prix des lecteurs Gallimard 2016, du 
Grand prix des lectrices de Elle 2017 et du Grand prix des lyciennes de Elle 2017.

" (...) Ce deuxième roman a été désigné dès le premier tour par les jurés du Goncourt, par six voix sur dix. Étaient également en lice l'autre qu'on adorait de Catherine Cusset (Gallimard), Petit Pays de Gaël Faye (Grasset) et Cannibales de Régis Jauffret (Seuil). (...) Avec deux nommés sur quatre, Gallimard avait mathématiquement une chance sur deux de l'importer» ${ }^{(1)}$

Notre choix du corpus sera porté sur le second roman Chanson Douce de Leila Slimani qui s'ouvre sur un choc terrible, celui du meurtre de deux enfants en bas âge, Mila et Adam, par leur nourrice Louise et sa tentative de se suicider. «(...)Chanson Douce s'inscrit dans la lignée cruelle de ces récits - des bonnes, de JeanGenet (1947) à Cérémonie de Claude Chabrol (1995)- inspirés par l'affaire des sœurs Papin, deux servantes qui avaient assassiné leurs patronnes en $1933 \ldots{ }^{(2)}$

Tiré d'un fait-divers, un double infanticide dans l'un des superbes quartiers de New York en 2012, Chanson Douce saisit les lecteurs avec une force surprenante tenant autant à la perfection de sa narration qu' à son style précis. 
«(...)Yoselyn Ortega, la "nounou tueuse", comme l'ont renommée les médias anglosaxons, a été reconnue coupable par la justice (...) et devrait être condamnée à la prison à perpétuité.» (3)

Ce fait divers indigne a inspiré à l'auteure Leila Slimani la trame de son roman à succès "Chanson Douce". Dans un entretien, la romancière déclare

«(...)c'est le fait divers qui m'a permis de décoller du réel. J'avais une idée théorique mais je n'arrivais pas à trouver l'enclencheur concret qui me permettrait de trouver le rythme narratif. J'ai lu ce drame dans Paris Match. Il entrait en résonance avec d'autres obsessions ${ }^{(4)}$

En plus elle ajoute

"Je me souviens très bien, dans le journal, de sa photo dans la bibliothèque de l'appartement, avec les parents qui disaient:" Elle faisait partie de la famille!" Et puis, un jour, la mère a trouvé les pièces plongées dans le noir et les enfants assassinés par le nounou qui avait tenté de se donner la mort. L'écriture est partie de là »(5)

Le titre du roman Chanson Douce est un titre accrocheur. Au premier contact avec ce titre, nous lecteurs, nous nous attendons à un conte magique ou à une histoire paisible ou plutôt à la mélodie d'Henri Salvador, la berceuse dans

(La Sociopathie de «Louise » dans Chanson Douce) Dr. Abir Chéhata 


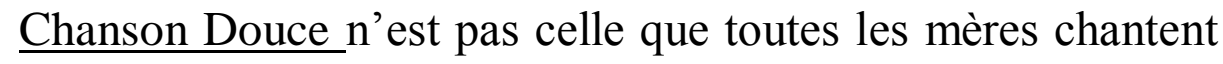
, elle suscite au contraire la terreur et laisse en l'âme une impression funèbre et triste.

Il en est ainsi la définition qu' en propose Charles Grivel :

"Si lire le roman est réellement le déchiffrement d'un fictif secret constitué puis résorbé par le récit même, alors le titre, toujours équivoque et mystérieux, est ce signe par lequel le livre s'ouvre : la question romanesque se trouve dès lors posée, l'horizon de lecture désigné, la réponse promise. Dès le titre l'ignorance et l'exigence de son résorbement simultanément s'impose. ${ }^{(6)}$

Nous lecteurs, nous sommes embarrassés par la non correspondance du contenu du roman au titre. D'après Gérard Genette, il est question d'un titre antiphrastique. Or « les titres antiphrastiques, enfin, présentent ironiquement le contenu du texte. La joie de vivre, dont la protagoniste est obsédée par la hantise de la mort, est le titre d'un des romans les plus noirs de Zola.» ${ }^{(7)}$

Chanson Douce, est donc un titre antiphrastique vu l'évidente contradiction entre le titre et le contenu dramatique dans lequel s'inscrit le roman de Leila Slimani. L'incipit met les lecteurs immédiatement au sein de l'action ainsi que les événements, à cet égard, on peut déclarer que l'incipit est ce qu'on appelle un «incipit in medias-res» qui est défini comme tel «incipit narratif qui réalise une entrée directe dans

(La Sociopathie de « Louise » dans Chanson Douce) Dr. Abir Chéhata 
l'histoire sans aucun élément introductif explicite et qui produit un effet de dramatisation.» ${ }^{(8)}$

À travers ce titre, la romancière saisit l'attention des lecteurs dès le début du roman jusqu'à son point final, les bouleversant par l'effet de proximité de ce qu'elle raconte.

À partir du dénouement "Le bébé est mort ${ }^{(9)}$ l'auteure remonte le temps et explore le personnage de la nounou apparemment idéale dont le trouble grandit tout au long du roman, nourri par un sentiment d'inaptitude et de rancune à l'égard de la vie.

Myriam Massé est une femme épuisée par une vie trop étriquée, qui ne lui convient pas. Avec ses deux enfants, cette vie est devenue de plus en plus compliquée: faire les courses, faire le ménage, donner le bain et aller chez le médecin. Myriam en a assez de continuer, elle s'est sentie enfermée dans son rôle de mère au foyer qu'elle détestait. De même, elle s'est sentie mourir dans "Ce bonheur simple, muet, carcéral, [qui] ne suffisait pas à la consoler ${ }^{(10)}$

Quant à Paul Massé, il est engagé comme producteur d'artistes dans un studio célèbre où il passait ses matinées et ses nuits.

«Cette vie de cocon, loin du monde et des autres, les protégeait de tout.» ${ }^{(1)}$

Soudainement, Myriam décide de reprendre sa carrière au sein d'un cabinet d'avocats dirigé par son ancien camarade de la faculté de droit.

(La Sociopathie de « Louise » dans Chanson Douce) Dr. Abir Chéhata 
Alors, le jeune couple parisien s'est mis à la recherche en urgence d'une nounou parfaite à domicile. Tâche qui s'annonce compliquée à Paris.

Le couple Paul et Myriam ne veut pas d'une nounou ayant encore de petits enfants, elle doit être disponible. Les auditions se poursuivent : une nourrice Philippine "Gigi" est arrivée en retard en murmurant des excuses, elle s'exprimait très mal en français, ce qui mène alors Paul à lui parler en anglais. Une nourrice Ivoirienne "Caroline", sans papiers passant l'entretien à se plaindre de son mal de dos et de ses problèmes de circulation et une nourrice marocaine "Malika" qui se mettait à parler en arabe avec Myriam, craignant par là de créer une complicité sous-entendue au nom «de la solidarité d'immigrés ».

Mais Myriam s' appuie sur le fait de refuser les nounous sans papiers en focalisant les dangers d'embaucher une personne de ce type.

À ce propos, la romancière dit :

«Pas de sans-papiers, on est d'accord! Pour la femme de ménage ou le peintre, ça ne me dérange pas. Il faut bien que ces gens travaillent, mais pour garder les petits, c'est trop dangereux. » (12)

Après ce casting ferme, le couple engage Louise qui, à son tour, conquiert rapidement l'affection des petits enfants, captive les parents et occupe peu à peu, une place primordiale dans le foyer en comblant leurs fantasmes:

(La Sociopathie de « Louise » dans Chanson Douce) Dr. Abir Chéhata 
enfants bien peignés, dîner préparé et ménage «tenu au cordeau»(13).

Petit à petit, un fossé s'élargit entre le couple et la nounou à cause de petits incidents successifs : par exemple quand elle maquille à l'excès Milla, fille de quatre ans ou quand elle refuse le gaspillage des aliments périmés au point d'en faire nourrir les enfants.

Par conséquent, le réconfort senti auparavant s'anéantit et perd sa vigueur.

De moins en moins à l'aise, le couple ne parvient pas à se débarrasser d'elle jusqu'au jour du crime.

"Bien sûr, il suffirait d'y mettre fin, de tout arrêter là. Mais Louise a les clés de chez eux, elle sait tout, elle s'est incrustée dans leur vie si profondément qu'elle semble maintenant impossible à déloger...» ${ }^{(14)}$

Par ailleurs, notre écrivaine s'est inspirée identiquement de son expérience personnelle. Lors d'un entretien, elle proclame :

"J'ai toujours été fascinée par la relation très étrange, très ambiguë qui se noue avec les nourrices [...]. Quand j'étais petite, nous avions des nounous à la maison et j'étais déjà sensible à la position assez cruelle de ces femmes qui nous élevaient comme des secondes mères qui restaient, invariablement, des étrangères. Et puis, j'ai moi-même 
engagé une nounou pour s'occuper de mon fils, et j'ai découvert ce monde de la "garde d'enfant" et son organisation économique et sociologique. "(15)

D'après Xavier Garnier, le personnage dans le roman possède le rôle de «faire avancer l'intrigue, révéler un monde ou [ne servir] à rien» ${ }^{(16)}$. Selon son intérêt, le personnage se définit donc comme «un moteur, une conscience ou un parasite» ${ }^{\text {(17). }}$

Plus le personnage est étrange, mystérieux plus il captive, attire l'attention en suscitant la curiosité . cela nous presse à nous concerner de plus près du cas de la nounou Louise en expliquant sa sociopathie et en le reliant à des figures mythiques.

\section{Portrait de la nounou "Louise"}

Le portrait est un aspect particulier de la description permettant à l'écrivain de présenter ses personnages.

Selon Philippe Hamon, le personnage représente la somme de ses caractéristiques à savoir son nom, le portrait physique, son identité ainsi que les différentes apparences que la romancière lui accorde. Elle nous renseigne donc sur son passé, sa vie et son rang social.

De même, en se rapportant à l'analyse faite par Philippe Hamon à propos du portrait physique du personnage, Vincent Jouve note :

" Le portrait du personnage passe d'abord par la référence au corps. Ce dernier peut être beau, laid, déformé, humain, non-humain. Le portrait, instrument essentiel de la caractérisation du

(La Sociopathie de « Louise » dans Chanson Douce) Dr. Abir Chéhata 


\section{personnage, participe logiquement à son évolution. "(18)}

Nous remarquons que Leila Slimani a exposé longuement le style vestimentaire de la nounou. Elle décrit son chemisier à col claudine, sa longue jupe dont Louise prend un grand soin, ce qui révèle un caractère strict. De plus, l' écrivaine détaille les chaussures de la nounou en se préparant pour aller chez les Massé, le premier jour du travail. Louise s'intéresse souvent à son apparence, elle veut se montrer élégante malgré le peu d'argent qu'elle possède or, l'usage habituel des choses démodées manifeste la vie économe et rude de Louise.

"Elle a délicatement étalé sur le canapé sa jupe longue et son chemisier. Elle attrape les ballerines qu'elle a posées par terre, un modèle acheté il y a plus de dix ans (....)(....) elle a fait teindre ses cheveux chez le coiffeur. (...) elle se maquille et le fard à paupières bleu la vieillit, elle dont la silhouette est si frêle, si menue, que de loin on lui donnerait à peine vingt ans. " ${ }^{(19)}$

Le physique peut nous renseigner sur 1' âge de la personne, mais il n'est pas certain. Aussi, le corps humain peut-t-il avoir une apparence qui apparaît bien plus jeune ou plus vieux que la vérité selon son état physique ou son style de vie.

Louise rassure les Massé par ses apparences. Ils admirent sa bonté qui masque derrière elle une figure mystérieuse.

«(...) Elle a le regard d'une femme qui peut tout

(La Sociopathie de « Louise » dans Chanson Douce) Dr. Abir Chéhata 
entendre et tout pardonner. Son visage est comme une mer paisible, dont personne ne pourrait soupçonner les abysses. " ${ }^{(20)}$

De plus, le nom du personnage joue un rôle primordial, il porte une signification particulière c'est pourquoi quelques patronymes donnent un indice tantôt sur le caractère tantôt sur la condition sociale du personnage.

"Louise" est un prénom d'origine allemande «Hlodowig », dont la signification divisée en deux morphèmes «hold» un adjectif qualificatif signifiant «glorieux» ou «illustre » et «wig » un nom désignant «bataille » ou « combattant ». Il résulte que Louise représente une femme combattante et glorieuse comme l'assure Leila Slimani

" Elle observe chaque pièce avec l'aplomb d'un général devant une terre à conquérir.»(21)

Dans les semaines qui ont suivi son arrivée au foyer du couple Parisien, elle se montre une femme de ménage perfectionniste imposant ses manières ainsi que "son goût pour la perfection.» ${ }^{(22)}$. Louise recoud les boutons de leurs vestes ; elle refait les ourlets des jupes et des pantalons, elle accroche des sachets de lavande entre les manteaux.

Quant aux enfants, Louise note les horaires de la danse, des sorties à 1 école, des rendez-vous chez le pédiatre. De même, elle copie le nom des médicaments que prennent les petits. Le personnage de Louise apparaît très mystifié, Leila Slimani nous peint une nounou parfaite en disant :

(La Sociopathie de «Louise » dans Chanson Douce) Dr. Abir Chéhata 
"Le couple rit, incrédule, de cette nouvelle vie qui est la leur. Ils ont le sentiment d'avoir trouvé la perle rare, d'être bénis. "(23)

Aussi, elle est comparée à une fée, un être formidable qui ne s'arrête pas de lutter pour vivre. Elle cherche à gagner l'attention et l'admiration en vue de garantir son salaire.

Voulant être à la hauteur de l'image d'une vie exemplaire, le couple superficiel est devenu complètement dévoré par l'ambition professionnelle. Nous trouvons Myriam, très occupée par son travail, elle a plus de conversations entre collègues, elle a plus de dossiers, entre les mains à étudier par cœur et elle passe des journées au tribunal à défendre ses clients avec flamme.

Quant à Paul, il est dans son studio en train de travailler sur ses propres sons. À la connaissance d'un chanteur connu : tous les deux parlent pendant des heures et des heures de leurs goûts communs, des arrangements qu'ils souhaitent et le chanteur finit par lui proposer de réaliser son prochain disque.

Mais comme affirme Leila Slimani

"Une famille c'est l'ensemble uni que forment les parents et leur enfant. [...] On est simultanément parent et enfant. Le parent produit l'enfant, l'enfant produit le parent, pas l'un sans l'autre! On «est » famille parce qu'on "naît» ensemble. La famille est chargée de mystère parce que c'est "là qu'on naît », avant d'y grandir.

(La Sociopathie de « Louise » dans Chanson Douce) Dr. Abir Chéhata 
Cette définition [représentant] la famille comme une matrice (...) d'où sortirait chacun de ses membres. »(24)

Rapidement, Louise est devenue indispensable. Elle ne compte plus ses heures et fait beaucoup plus que ce qu'on attendait d'elle. Le couple trouve qu'en plus d' être une bonne nourrice, Louise est également cuisinière. Il commence à inviter des amis qui demandaient des banquettes de veau, des pots-au-feu, des jarrets à la sauge et de légumes croquants que fait mitonner Louise. Alors, Myriam avoue souvent : "C C'est ma nounou qui a tout fait.» ${ }^{(25)}$

Pierre Daco définit le perfectionnisme de Louise comme "un besoin permanent poussant l'infériorité à rechercher une apparence de perfection; cette recherche s'accompagne d'obsession diffuse, ou forte, ou angoissée » (26)

Leila signale le complexe d'infériorité dont la nounou souffre cela traduit son obsession pour la perfection. Louise donne souvent plus pour combler ce sentiment.

"(...) Louise fait de cet appartement brouillon un parfait intérieur bourgeois. Elle impose ses manières désuètes, son goût pour la perfection.» ${ }^{(27)}$

Derrière cette apparence se cache quelque chose de terrible. Louise paraît être tendre et docile mais si on la contrarie, elle peut exhiber ses griffes en changeant le visage. Or,il faut se méfier des personnes qui réclament trop de bien, cela implique de savoir lire entre les lignes pour découvrir le problème.

(La Sociopathie de « Louise » dans Chanson Douce) Dr. Abir Chéhata 


\section{La Sociopathie de la nounou "Louise"}

Selon Liliane Holstein "sous [le] masque de normalité [ des sociopathes], [ils sont] incapables de fonder une famille, de $s$ ' entourer d'amis solides, ils seraient désespérément isolés sur le plan affectif. " (28)

La nounou Louise est une sociopathe c'est-à-dire une personne affectée par des troubles de la personnalité manifestant un comportement antisocial, irritable et dépourvu de culpabilité.

La romancière signale que Louise s'échappe de tout le monde, elle n'a ni voisins, ni amis. Dans le jardin, elle tient à mettre de la distance avec les autres nourrices en refusant tout lien avec autrui.

" Face à Louise et à son silence, Wafa parle comme on se confie à un prêtre ou à la police ${ }^{(29)}$

D'après la définition de Larousse «La sociopathie est un trouble de la personnalité, c'est-à-dire qu'il s'agit d'un ensemble de troubles dont les symptômes sont des traits de personnalité stables et permanents . Ce caractère rigide et" mal adapté "induit des comportements dysfonctionnels dans la vie en société ainsi qu'une souffrance pour la personne ${ }^{(30)}$

La romancière Leila Slimani mentionne de petits éléments sur la personnalité de Louise qui conduiront peu à peu au doute et au soupçon. Elle nous indique que qu'elle est un personnage troublé, complexe et ambigu dissimulant un caractère mélancolique par exemple, la couche de fard mauve mise sur ses yeux sert à cacher ses défauts et son choix de la couleur violette signifie son sentiment de mélancolie.

(La Sociopathie de « Louise » dans Chanson Douce) Dr. Abir Chéhata 
La plupart des gens mélancoliques prétendent dissimuler leur souffrance et préfèrent l'intérioriser plutôt que la montrer aux autres, ce qui ne mène qu'ajouter ce défi.

Quant aux caractéristiques de la mélancolie ,Freud marque : «Psychiquement, mélancolie se caractérise par une humeur profondément douloureuse, un désintérêt pour le monde extérieur, la perte de la faculté d'amour[et] l'inhibition de toute activité... » ${ }^{(31)}$

Ces symptômes n'apparaissent pas souvent en même temps, Freud pense qu'ils se manifestent tous à un moment précis au cours d'un état mélancolique.

Notre protagoniste Louise porte en elle un côté noir, obscur cela se traduit par les histoires qu'elle raconte sans aucun effort aux enfants, ces histories horribles dont les personnages se répètent le plus souvent en répandant de la frayeur.

" Des orphelins, des petites filles perdues, des princesses prisonnières et des châteaux que des ogres terribles laissent à l'abandon.(..) D'où viennent ces histoires? Elles émanent d'elle, en flot continu... »(32) Toujours, la nounou se révèle plongée dans la tristesse et la mélancolie comme la déclare cette phrase

" la matinée est gachée par la mélancolie de louise et à table, sur la terrasse de la petite taverne, personne ne parle. "(33)

Pierre Daco nous livre d'autres signes identifiant ce caractère mélancolique en déclarant qu' "Il se présente chez le médecin avec un tas de "petits papiers" où il a noté des multiples troubles qui le font souffrir. »(34)

(La Sociopathie de « Louise » dans Chanson Douce) Dr. Abir Chéhata 
En plus

"(...) la mélancolie que nous connaissons tous à des moments divers de l'existence peut s'installer durablement et gravement.

Elle prend alors la forme d'une dépression maladive. Sa victime éprouve une douleur morale intense, (...) un sentiment de culpabilité et d'autodépréciation. (...)Des troubles physiologiques: ralentissement psychomoteur pouvant atteindre l'inertie, insomnies (...) aggravent souvent son mal. »(35)

Selon cette définition, la mélancolie représente une double sensation forte de tristesse et de dépression morale. Tel est le cas de la nounou Louise qui a noté dans un petit carnet à la couverture fleurie le terme "Mélancolie délirante ${ }^{(36)}$ utilisé par un médecin lors de son séjour à l'hôpital HenriMondor Comme l'annonce la romancière "une brutale mélancolie étreint Louise.... ${ }^{(37)}$. Or, Cette mélancolie accompagnée d'une solitude déchirante qui s'allonge de plus en plus de façon à devenir une sorte d'escapade des problèmes familiaux et financiers pesant sur sa vie avec le couple.

«Mélancolie délirante ».

Louise avait trouvé ça beau et dans sa tristesse s'était subitement introduite une touche de poésie, une évasion. ${ }^{(38)}$

La solitude dévore Louise dans un petit studio pitoyable de Créteil, en périphérie de la ville qu'elle n'arrive jamais à payer après la mort de son mari Jaques lui laissant un tas de dettes ainsi que la fuite de sa fille illégitime

(La Sociopathie de « Louise » dans Chanson Douce) Dr. Abir Chéhata 
Stéphanie. Seule, elle n'a qu'à regarder l'émission Enquêtes criminelles devant sa télévision qui lui tient compte.

Solitude se définit «État de quelqu'un qui est seul momentanément ou habituellement» ${ }^{(39)}$

D'après cette définition, la solitude signifie n'avoir aucun rapport social avec autrui. Elle ne représente pas seulement une sensation d'isolement mais c'est le fait de sentir qu'il n'existe personne proche or voici quelques paragraphes du récit reprenant ce thème de la solitude de Louise.

«La solitude agissait comme une drogue dont elle n'était pas sûre de vouloir se passer.»(40)

Ici la nounou sent la solitude comme une drogue telle qu'une dose nécessaire qu'elle est obligée à prendre au fil des mois passés chez le couple et qu'elle ne peut s'en dépasser.

De même, Louise reste constamment retirée au square où les enfants Mila et Adam jouent, ne laissant personne l'atteindre. C'est comme si elle portait sur elle un voile qui la couvre et la préserve de l'avidité des gens .Elle ne leur donne aucune chance de la pénétrer.

«(..) les regards appuyés(..) la gênent. Elle déteste cette sensation d'être épiée, prise au piège. » ${ }^{(41)}$

En outre, cette solitude

« qui collait à sa chair, à ses vêtements, a commencé à modeler ses traits et lui a donné des gestes de petite vieille ${ }^{(42)}$

(La Sociopathie de «Louise » dans Chanson Douce) Dr. Abir Chéhata 
Même avant la mort de son mari, Louise n'a jamais compté sur lui, en plus, il n'a cessé de l'humilier en disant «Je ne suis pas comme toi, (...) je n'ai pas une âme de carpette, à ramasser la merde et le vomi des mioches. Il n'y a plus que les négresses pour faire un travail pareil.»(43)

Quant à sa fille Stéphanie, elle sort toute la nuit et ne rentre qu'au matin, se jette sur le frigidaire sans s'asseoir. Une fois, elle s'est teinte les cheveux en rouge. "Elle s'est mise à disparaître, des week-ends entiers. Et puis un jour, elle n'est pas revenue. Plus rien ne la retenait.

(...) Ni le lycée, qu'elle avait quitté depuis longtemps. Ni Louise.»(44)

Louise éprouve toujours une solitude perçante, poignante qui a laissé son empreinte sur son comportement bizarre, singulier et étonnant surtout après la mort de Jacques et la fugue de sa fille Stéphanie. Ainsi, elle est devenue seule sans personne autour d'elle.

Dans une confession, la romancière déclare que son adolescence est imprégnée d'une solitude hibernale pendant son séjour à Paris pour les études.

"Cela a été très dur, j'avais 17 ans. Je ne me rendais pas compte que j'allais connaître une telle solitude. Je me souviens de semaines entières où je ne parlais à personne en dehors des cours. Les Parisiens prennent un café le soir ensemble et, après, chacun rentre dîner chez soi. C'est inimaginable au Maroc, où on invite les

(La Sociopathie de «Louise » dans Chanson Douce) Dr. Abir Chéhata 
gens qu'on sait seuls. Le premier hiver a été interminable, $11^{(45)}$

Avec toutes ces pires circonstances secrètes de Louise, celle ci est au courant de tout ce qui concerne le couple Mariam et Paul mais le couple, au contraire ignore tout d'elle.

Ajoutons encore que la romancière ne détermine pas la nounou par un nom bien propre à elle. Celle-ci est connue sous le prénom de Louise. L'auteure n'annonce ni sa famille ni ses origines. Cet être demeure si ambigu et si étrange. À cet égard, les antécédents de Louise sont presque inconnus, son passé est ignoré cela explique son mystère.

«Paul et Myriam sont séduits par Louise, par ses traits lisses, son sourire franc, ses lèvres qui ne tremblent pas. Elle semble imperturbable. Elle a le regard d'une femme qui peut tout entendre et tout pardonner.» ${ }^{(46)}$

Cette chère Louise, à toute force, indispensable faisant partie de la famille, mais qui, réellement, demeure une étrangère malgré son dévouement corps et âme sans intérêt dans le quotidien des Massé.

Malgré l'intelligence et l'ouverture d'esprit du couple, il exagère de plus en plus à épuiser Louise par plus de tâches sans penser au moins résoudre l' un de ses problèmes infinis qui la rend une femme effacée.

$$
\begin{aligned}
& \text { "(...) Elle bosse pour qu'on } \\
& \text { puisse bosser. » (47) }
\end{aligned}
$$

L'arrivée d'une lettre du Trésor Public au couple lui demandant de saisir sur le salaire de Louise la somme qu'elle 
lui doit car elle n'a jamais répondu « à aucune lettre de relance "(48)

invoquant l'histoire de changement d'adresse, rejetant la faute sur son mari qui était si prudent, Louise a nié la réalité.

«(...) elle devait faire la morte, elle qui de toute façon ne représente rien, ne possède rien. Qu'est-ce que ça peut leur faire? Qu'ont-ils besoin de la traquer ?»(49)

Nous lecteurs, nous sommes témoins des cas de mélancolie, de solitude dans la vie de Louise. Elle a indéfiniment accepté d'exécuter tous les ordres proposés par le couple pour combler le vide de sa vie.

La toile du roman se tisse, saignante et aveuglante : du simple secours de nounou, Louise devient utile, dynamique ensuite indispensable.

Myriam a senti que la présence de cette nounou devenait gênante, elle a pris trop de place, elle s'est permis des ingérences.

De gênante à pesante, l'existence de Louise bouleverse l'équilibre du couple mais sans guère l'agiter. Le couple commence à regarder Louise de travers. Ses gestes les indisposent de plus en plus.

"le couple passe d'une adoration enthousiaste à une antipathie viscérale-sans jamais- par confort, gêne et faiblesse - oser licencier Louise» ${ }^{(50)}$

Après avoir passé deux nuits d'angoisse, couchée, malade dans son appartement aux vitres sales «[Louise] se sent prête à reprendre le travail.»( 51 ) comme le prouve 
Freud "l'angoisse est un affect. C'est dans le corps qu'elle est ressentie (...) elle est un quantum d'énergie libidinale qui n'a pu parvenir à la décharge faute d'avoir pu trouver une voie libératrice. À défaut de pouvoir se lier à une représentation acceptable pour se frayer un passage vers la satisfaction, cette énergie s'accumule et procuré un désagrément ressenti dans le corps. »(52)

Notons aussi que cette angoisse accompagnée «d'une vieille morbide, (..) est devenu[e] une obsession.» ${ }^{(53)}$

Or l'angoisse

"est un état psychologique voisin de l'anxiété.

$L$ 'angoisse est une manifestation profonde d'inquiétude.

Elle peut survenir de manière ponctuelle.........» ${ }^{(54)}$

De même, la romancière a souffert, elle aussi, d'une mélancolie et d'une angoisse depuis son adolescence dans sa ville natale, Rabat, elle avoue :

"A quinze ans, je broie du noir. La vie n'a pas de sens, je suis jeune et déjà lasse. Je m'apitoie sur mon sort, j'écoute des chansons tristes, je pleure dans ma glace. Ma mère me demande ce que j'ai. Je lui réponds que je n'en sais rien, que j'ai des angoisses, que j'ai peur. "Mais peur de quoi ? », me questionne-t-elle. C'est bien le problème, je ne sais pas. (...) Au milieu 
d'une conversation, elle glisse le nom du mal dont je souffre. Elle le prononce doucement, avec tendresse et ironie; elle dit que c'est de la mélancolie.»(55)

Louise nourrit de profonds ressentiments contre Myriam, la mère des deux enfants et le style de vie aisé du couple qui contrarie avec la pauvreté dans laquelle elle a vécu longtemps.

"Une haine monte en elle. (...) une haine qui brouille tout. Elle est absorbée dans un rêve triste et confus.»(56)

Louise éprouve le sentiment d'être rejetée de cette famille, d'avoir éprouvé qu'elle est sur le bord de perdre le peu qu'elle possède: une famille d'adoption et de suite une raison d'exister.

Chaque personne semble avoir une demeure où se rendre, Louise, n'a jamais eu ni une maison ni même une chambre à elle.

"Elle marchait dans la rue comme un décor de cinéma dont elle aurait été absente, spectatrice invisible du mouvement des hommes. Tout le monde semblait avoir quelque part où aller.»(57)

En effet, Louise n'a qu'une envie : Trouver un toit, s'y loger, creuser un nid chaud, faire partie d'une famille et ainsi appartenir à quelqu'un. Or, la vue d'un homme qui chie sans honte dans la rue devant la porte de son étroit appartement miteux où la douche pourrie s'est effondrée la fait penser à son propre avenir inquiétant.

(La Sociopathie de « Louise » dans Chanson Douce) Dr. Abir Chéhata 
la seule solution de Louise consiste à avoir un nouveau-né c'est-à-dire que Myriam tombe enceinte pour la troisième fois.

«Un nourrisson qui les tiendrait tout près les uns des autres ,qui les lierait tout près les uns des autres, qui lierait dans un même élan de tendresse ${ }^{(58)}$

Cette nouvelle grossesse est son dernier espoir pour garder sa place chez le quotidien bourgeois. Elle a une persuasion brûlante, torride que son bonheur leur concerne. Qu'elle est à eux et qu' ils sont à elle.

«Le lendemain, elle fait avec plus de soin que d'habitude le lit de Paul et de Myriam. Elle passe sa main sur les draps, elle cherche une trace de leurs étreintes, une trace de l'enfant dont elle est sûre à présent qu'il est à venir ${ }^{(59)}$

Un jour, Louise pousse Mila à demander à Myriam si elle porte un bébé dans son ventre. "oh non, plutôt mourir» ${ }^{(60)}$ a répondu Myriam en riant. À chaque tentative de la part de Louise, un mur épais s'élève devant elle l'empêchant à tout jamais de vivre comme n'importe quel être humain sans besoin.

« (...) L’inégalité ressemble à une bombe à retardement. Les petits déclencheurs de terrorisme agitent cet esprit délirant, que l'insécurité, masquée sous sa méticulosité, exhibera en accéléré.» ${ }^{(61)}$ 
L'atmosphère au sein du quotidien des Massé est devenue de plus en plus pesante, Leila Slimani nous entraîne dans une progression dramatique si inévitable. Des idées absurdes, incongrues la hantent or elle se laisse, descendre, peu à peu aux abîmes, dans les méandres de la folie.

«Enfermée dans l'appartement des Massé, elle a parfois l'impression de devenir folle.» ${ }^{(62)}$

Il est temps d'évoquer "par exemple, M.Plaza cité par J.P Baranger[qui] définit(...) la folie comme le « rapport d'antagonisme qui se noue entre les positions d'un individu et le système d'intelligibilité $d u$ groupe auquel il

appartient ${ }^{»(63)}$

Nous pouvons conclure que Louise est frappée par les symptômes de la schizophrénie puisqu' elle souffre de trouble de comportement comme le montre en évidence l'incident où Myriam lui annonce qu'ils vont partir en vacances à la montagne chez les parents de Paul, celle-ci ne se fâche plus pour montrer sa frustration.

\section{En outre}

«La folie est un terme très général englobant un ensemble de troubles mentaux distincts. La schizophrénie, les troubles bipolaires ou encore la paranoïa en font par exemple partie.

La folie peut ainsi prendre de multiples formes et s'exprimer de bien de façons. Globalement, on considère qu'il s'agit d'un comportement 
anormal, pouvant parfois être source de danger

pour le malade et/ou les autres personnes.» ${ }^{(64)}$

Des refrains funestes bercent Louise pendant sa marche. Des phrases incompréhensives habitent son esprit.

" Il faut que quelqu'un meure. Il faut que

quelqu'un meure pour que nous soyons

heureux. ${ }^{(65)}$

À ce stade, nous lecteurs, avons compris comment la vie de Louise ainsi que ses problèmes interminables ont contribué à l'effacer définitivement cette femme. C'est ce que Leila Slimani explique clairement en disant :

"Son cour s'est durci. Les années l'ont recouvert d'une écorce épaisse et froide. (...) Plus rien ne parvient à l'émouvoir (...)Elle a épuisé tout ce que son cour contenait de tendresse. "(66)

Dans Le Petit Larousse illustré de 1998, la violence se définit : "caractère de ce qui se manifeste, se produit ou produit des effets avec une force intense, extrême, brutale [...] extrême véhémence, outrance des propos, du comportement » 67 ) Encore, d'après la philosophie de Blandine Kriegel, la violence est "la force déréglée qui porte atteinte à l'intégrité physique ou psychique pour mettre en cause dans un but de domination ou de destruction l'humanité de l'individu. » la violence est [donc] opposée à un usage contrôlé, légitime et mesuré de la force. "(68)

Loin du regard du couple, nous lecteurs, avons assisté à cette plongée abyssale vers une folie dont nous seuls connaissons

(La Sociopathie de « Louise » dans Chanson Douce) Dr. Abir Chéhata 
la fin: le meurtre des deux enfants. Cet acte ignoble et impardonnable commis avec du sang froid.

Après deux mois d'enquête menée par le capitaine Nina Dorval, celle-ci décide nettement de déguiser le rôle de la nounou visant à obtenir plus d'informations et plus d'explications.

"Nina a prévenu le juge d'instruction, le procureur, les avocats. "C'est moi a-t-elle dit, qui ferait la nounou. Personne n'oserait la contredire. Le capitaine connaît cette affaire mieux que personne. "(69) 


\section{Conclusion}

Chanson Douce se classe parmi les œuvres modernes qui palpent différents genres littéraires « polar, thriller, psychologie....», à travers elle, la romancière témoigne plusieurs thèmes actuels ainsi qu'universels dont la maternité, l' éducation, le rapport entre employeurs et employés mais elle insiste en particulier sur 1' impact que peut avoir le statut social et psychologique sur une personne. Sans pathos, ce roman noir est écrit avec des mots crus, abrupts mais dont émane une certaine douceur évoquant l'histoire d'une mère qui culpabilise de retourner travailler, de confier ses enfants à une inconnue et d'un père souvent absent toujours occupé par son travail. Tous les deux sont ambitieux et ceci les fait éloigner d'un quotidien qui s'englue autour de la nounou Louise, dévouée, active, omniprésente mais discrète.

La rapide dépendance du couple vis-à-vis de leur nounou est dépeinte avec subtilité, les personnages ordinaires qui vivent une vie simple se trouvent au prise avec une sociopathe.

Leila Slimani indique les attitudes complexes qui peuvent provoquer des conséquences funestes, c'est-à-dire, elle concrétise tous ces actes dans son personnage romanesque de Louise . La romancière lui donne un rôle de nounou. Cette dernière a une présence intime mais jamais familière.Dans ce roman, Leila slimani pose la nounou au centre de l' intérêt. Dans Chanson Douce, elle nous parle de la puissance dévastatrice de cet exemple de réussite professionnelle qui perd complètement son équilibre. Elle décrit aussi la relation

(La Sociopathie de « Louise » dans Chanson Douce) Dr. Abir Chéhata 
entre les parents Myriam et Paul et la baby-sitter, louise durant laquelle il faudra mettre des limites.

$\mathrm{Au}$ fil des pages, nous ne pouvons pas nous empêcher de juger que ces parents enchaînés dans un style de vie débordé paraissent avoir éteint leurs pleurs intérieurs pour laisser survenir le pire.

\section{«(...) (...) on aimerait secouer Paul et} Myriam qui délèguent tout à Louise par fainéantise. Crier à Myriam que ses doutes ne sont pas infondés et qu'elle a raison de trouver la personnalité de sa nounou inquiétante. Renvoyer Louise chez elle lorsqu'elle fait sournoise !» ${ }^{(70}$

Effectivement, nous assurons que ces parents profitent de leur nounou tant qu'ils n'ont guère le temps de poser la question s'ils ne lui en font plus faire trop. C'est vrai qu'elle a accompli plus qu'on lui demandait dans l'annonce. De même, elle était sous - payée (particulièrement en prenant compte du nombre d'heures qu'elle travaille) si non elle aurait pu payer au moins la location de son studio médiocre, ne dissipant que très peu pour se nourrir et s'habiller. Louise a accumulé tout un tas d'angoisses, de souffrances, de colères refoulées pour arriver à un acte ignoble celui de tuer les enfants qu'elle adorait. Myriam et Paul ne parviennent plus à s'emparer de la dimension de la désintégration de leur nounou, Louise, en lui confiant les êtres qui comptent les plus chers pour eux .

$\breve{A}$ vrai dire,plusieurs facteurs tels que : la misère, la pauvreté et les dettes ont contribué au crime mais ces éléments ne 
pardonnent guère 1' acte atroce qui est accompli. Nous lecteurs, nous ne pouvons pas être insensibles au personnage de Louise, nous ressentons de la pitié, de l' amertume, de la tristesse mais aussi nous sommes de même choqués, révoltés, nous ne parvenons pas à prendre un jugement précis.

Ce roman appelle toutes les mères ainsi que tous les pères même si beaucoup de nourrices sont sans aucun doute des personnes sensibles, responsables et professionnelles, il reste souvent l'éternelle question posée sans réponse : que se déroule-t-il une fois la porte refermée ?

Chanson Douce va plus loin de relater un drame, il expose une réelle réflexion sociale ainsi que psychologique complexes. Cette histoire est une histoire terrifiante, haletante au style incisif telle qu'une lame à l'image de son thème, une histoire réaliste tellement contemporaine parce qu'elle aborde le statut des nourrices, ébranle les parents et fera vivre d'une autre vision leurs relations avec leurs enfants. 


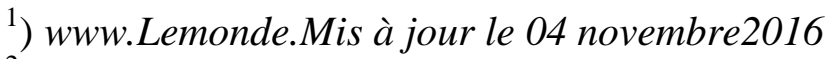

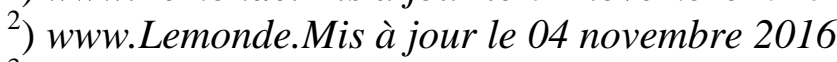

$\left.{ }^{3}\right)$ www.huffpostmaghreb.com 8/9/2019

4 ) https://www.letemps.ch/culture/2017/01/06leila-slimani-ne-realisetoujours-jai-recu-goncourt, consulté le 26 mars 2017

5) http://www.huffingtonpost.fr/2016/11/03/leila-slimani-goncourt-

2016-chanson-doucel, consulté le 6 février 2017.

6 ) Grivel (Charles), Production de l'intérêt, LaHaye-Paris, Mouton,1973, P.173

$\left.{ }^{7}\right)$ Genette (Gérard) cité par Vincent Jouve dans Poétique du roman, Ed.SEdEs, 1997, P.14

8) Del LunGo Andrea, "Pour une poétique de l'incipit» in Poétique $n^{\circ}$ 94 cité par Rullier - Theuret Françoise, Approche du roman, Hachette, P.58

9) Slimani (Leila), Chanson Douce, Éditions Gallimard 2016, P.13

10) Ibid., P.24

11) Ibid., P.19

12) Slimani (Leila), Chanson Douce, op.cit., P.16.

13) www.telerama.fr

14) Slimani (Leila), Chanson Douce, Op.cit., P.191

15 ) http://www.glamourparis.com/culture/livre-a-lire/articles/chansondouce-pourquoi-faut-il-absolument-lire-leila-slimani-/47089, consulté le 6 février 2017

16) Xavier,(Garnier), L'éclat de la figure, Éditions P.I.E.-Peter Lang, Bruxelles, 2002,p.9

17) Idem

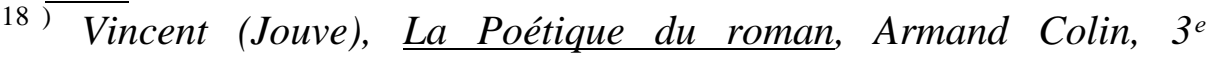
édition, 2010, p.85

19) Slimani (Leila), Chanson Douce, Op.cit., pp.33-34

${ }^{20}$ Ibid.,p.30

${ }^{21}$ Ibid., P.37

22) Ibid., P.38

23) $\underline{\text { Idem., }}$

${ }^{24)}$ https://www.Carin.info/revue-vie-sociale-et-traitements-2006-1page154.htm.

25 ) Slimani (Leila), Chanson Douce ,op.cit.,p.39 
${ }^{26}$ ) Daco (Pierre), Les prodigieuses victoires de la psychologie moderne, éditions Marbout,1960, p.79.

27 ) Slimani ( Leila), Chanson Douce, op.cit., pp.,37-38

28 ) https://www.20minutes.fr/sante/1342 149-20140403-manipulationcomment-reconnitre- sociopathe

${ }^{29}$ ) Slimani ( Leila), Chanson Douce, op.cit., p126.

${ }^{30}$ ) https://www.larousse.fr/dictionnairelfrançais/sociopathie/186766

31 ) Sigmund(Freud),Deuil et mélancolie Paris (Payots et

Rivages,2011[1917]),p45.

32 ) Slimani ( Leila), Chanson Douce, op.cit., pp41-42.

33 ) Ibid.,p81.

${ }^{2)}$ Daco (Pierre), Les prodigieuses victoires de la psychologie moderne, op.cit., P.338

${ }^{35)}$ https://www.psychologies.com/Daco-psycho/Melancolie

${ }^{36)}$ Slimani (Leila), Chanson Douce, op.cit., P.171

37 Ibid.,p226.

38) Slimani (Leila), Chanson Douce, op.cit., P.171

${ }^{39}$ ) https:www.Larousse.fr/dictionnaires/francais solitude/73340

${ }^{40}$ ) Slimani (Leila), Chanson Douce, op.cit., P.112

${ }^{41}$ Ibid., P.157.

42) Ibid., P.111

43) Ibid., P.107

44) Ibid. P.99

${ }^{45)}$ http://www.elle.fr/Loisirs/Livres/News/Leila-Slimani-rencontre-avecla-romanciere-de-l-ultramoderne-solitude-des-femmes-3142603

consulté le 24 mars 2017

46) Slimani (Leila), Chanson Douce, op.cit., P.30

47) Ibid ., P.16

${ }^{48)}$ Ibid., P. 162

49) Ibid., P.163

50) http://www.revue-etudes.com 8/9/2019

51) Slimani (Leila), Chanson Douce, op.cit., P. 172

${ }^{52}$ ) http://www..carin.info/revue-journal francais-de-psychiatrie-20064 page-40.htm.

${ }^{53)}$ Ibid., P.171 
${ }^{54)}$ https://sante-medecine.journaldesfemmes.fr/Faq/8092-angoissedefinition

${ }^{55}$ https://www.franceculture.fr/litterature/journee-de-la-languefrancaise-en-compagnie-de-leila-slimani, consulté le 22 mars 2017. ${ }^{56)}$ Slimani (Leila), Chanson Douce, op.cit., P. 171

${ }^{57)}$ Ibid., P. 112

${ }^{58}$, Ibid., P. 200.

59) Ibid., P.201

60) $\underline{\text { Idem }}$

${ }^{61}$ http://www.ledevoir.com/lire/483882/prix-goncourt-2016-unechanson-douce-qui-ne-l-est-pas

${ }^{62)}$ Slimani (Leila), Chanson Douce, op.cit., P. 122

${ }^{63}$ )cf Baranger(J.P), " Revendiquer l'asile » dans J. Garrabe, J.c.Pascal et coll Images de la psychiatrie,Paris,Frison-Roche,1996,pp373-377.

64 https://sante-medecine.journaldesfemmes.fr/Faq/17860-foliedefinition

${ }^{65)}$ Slimani (Leila), Chanson Douce, op.cit., P. 230

66) Idem

67) Le Petit La rousse Illustré, Paris, Editions Entièrement Nouvelle, 1998, P.1068

${ }^{68)}$ https://psychologie.savoir.fr/definition-violence/

${ }_{70}^{69}$ Slimani (Leila), Chanson Douce, op.cit., P. 237.

70 https://www.elle.fr/Loisirs/Livres/Prix-litteraire-deslectrices/Chanson-douce-de-Leila-Slimani-aux-editions-Gallimard$\underline{3414394}$ 


\section{Bibliographie}

\section{Corpus}

Slimani (Leila), Chanson Douce, Gallimard, Paris, 2016

\section{Ouvrages Généraux}

- Barthes, Roland, Poétique du récit, Seuil, Paris, 1977.

- Brunel, Pierre, La mythocritique : Théorie et Parcours, PuF, Paris, 1992.

- Daco, Pierre, Les Prodigieuses victoires de la psychologie moderne. Editions Marabout, 1960.

- Durant, Guilbert, Introduction à la mythologie : Mythes et sociétés, Éditions Albin Michel, Paris, 1996.

- Garnier, Xavier, L'éclat de la figure, Éditions P.I.E.- Peter Lang, Bruxelles, 2002.

- Genette, Gérard, Figures III, Seuil, Coll. poétique, Paris, 1972.

- Grivel, Charles, Production de l'intérêt, La Haye-Paris, Mouton, 1973.

- Jouve, Vincent, L'effet-personnage dans le roman, PuF, Paris, 1992. 
- Poétique du roman, Paris, Armand Colin, $3^{e}$ édition, 2010

- LEONARD-Roques, Véronique, figures mythiques : fabrique et métamorphoses, Presses université Blaise Pascal, Paris, 2008.

\section{Ouvrages psychanalytiques}

- Baranger(J.P), "Revendiquer l'asile " dans J. Garrabe, J.c.Pascal et coll Images de la psychiatrie, Paris, FrisonRoche, 1996

- Bukowski(c), contes de la folie ordinaire, Paris, Grasset, 1981. -Duprat(G.L), Les causes Sociales de la folie,Paris ,Alcan, 1900.

-Sicot(F), Maladie mentale et pauvreté,Paris, L'Harmattan, 2001.

- Sigmund(Freud), Deuil et mélancolie Paris (Payots et Rivages, 2011[1917]) 


\section{Articles}

- Del Lungo, Andrea, "Pour une poétique de l'incipit » in poétique no 94, avril 1993.

\section{Dictionnaires}

- Brunel, Pierre, Dictionnaire des Mythes littéraires, Éditions du Rocher, Paris, 2003.

- Chevalier, Jacques, Dictionnaire des symboles, Seghers, Paris, 1973.

- Guirland, Félix, SCHMIDT, Joêl (dir),Mythes

Mythologie :Histoire et dictionnaire Larousse , Paris , 1996.

- Le petit La rousse Illustré , Paris ,Edition Entièrement Nouvelle , 1998.

\section{Sitographie}

- www.lemonde mis à jour le 04 novembre 2016

- www.hulfpostmaghreb.com le 08/9/2019

- www.telerama.fr

- https://www.20minutes.fr/sante/1342 14920140403-manipulation-comment-reconnîtresociopathe 
- www.letemps.ch/culture/2017/01/06leila_Slimani_ne _realise_toujours_jairecu_goncourt consulté le 26 mars 2017

- www.huffingtonpost.fr/2016/11/03/leila_slimani_gonco urt_2016_ chanson-douce/ consulté le 6 février 2017

- http://www.glamourparis.com/culture/livre_alire/articles/chanson-douce-pourquoi-faut-ilabsolument-lire-leila-slimani/147089 consulté le 6 février 2017

- http://www.carin.info_revue_vie_sociale_et_traitemen ts_2006_1page_154htm .

- http://www..carin.info/revue-journal francais-depsychiatrie-2006-4 page-40.htm.

- https:/www.larousse.fr/dictionnaire/français/sociopath e/186766.

- http://www.psychologies.com/daco_psycho/Melancoli e 
- https://www.larousse.fr/dictionnaire//francais.solitude/ 73340

- https://www.sante_medecine.journaldesfemmes.fr/faq /8092_angoisse_ defintion

- http://www.revue-etudes.com 8/9/2019

- https://sante_medecine.journaldesfemmes.fr/faq/1786 0_folie_defintion

- https://psychologie_savoir.fr/definition_violence/

- https://www.franceculture.fr|litterature/journee_de_la_I angue_française_en_compagnie_de_leila_slimani, consulté le 22 mars 2017 .

- https://www.elle.fr/Loisirs/Livres/Prix-litteraire-deslectrices/Chanson-douce-de-Leila-Slimani-auxeditions-Gallimard-3414394

- https:/www.ledevoir.com/lire/483882/prix-goncourt2016-une-chanson-douce-qui-ne-I-est-pas

- https://www.elle.fr/Loisirs/Livres/News/leila_slimani_r encontre_avec_la_ 
romanciere_de_I_ultramoderne_solitude_des_femmes _3142603, consulté le 24 mars 2017. 
Table des matières

Introduction

p.3

Portrait de la nounou "Louise"

p.17

La sociopathie de la nounou Louise

p.26

Conclusion

p.52

(La Sociopathie de «Louise » dans Chanson Douce) Dr. Abir Chéhata 
الاعتلال الاجتماعي عند لويز في الأنشوده الهادئة

تبدأ رواية الأنشودة الهادئة بصدمة مخيفة مر عبة وهي موت طفلين حديثين العهد ميلان و آدم على يد مربيتهم لويز ومحاو لاتها الإنتحار. حاولنا في بحثنا أن نحلل عن قرب شخصية المربية لويز فهي كار هـه لوسطها الإجتمـاعي وهذا يعني أنها تعـاني من اضطر ابات في الثخصية حيث تُظهر تصرفات معاديـة للمجتمع .تخفي هذه الثخصية المعقدة و الغامضـة طـابع كئيب وحالـة الكآبـة هذه مصـطحبة بوحدة محزنـة وممزقـة تمتـد أكثر فـأكثر بطريقـة جعلتها كنوع من الهروب من مشاكلها الاجتماعية والمالية المُثقلة على حياتها مـع الزوجين.

تلازم لويز افكار محالة غير معقولتة وغير لائقة فهي تركت نفسها تنحدر شيئا فنثيئا نحو الهاوية في غيابات الجنون. الأنشوده الهادئة هي قصة حقيقية ومعاصره أيضـا لأنها تتطرق لحالة المربيات وتزعزع ثقه الآباء وتجعلهم يقيمون علاقاتهم مع أبنائهم برؤى مختلفة. 